\title{
EL MARKETING Y LA VENTAJA COMPETITIVA EN LOS ALUMNOS DE LA FCA - UNMSM (PERÚ), COMPARADA CON LOS ALUMNOS DE ADMINISTRACIÓN DE LA UNIVERSIDAD DE LOS ESTUDIOS DE BÉRGAMO (ITALIA)
}

\begin{abstract}
MARKETING AND COMPETITIVE ADVANTAGE IN THE STUDENTS OF THE FCA - UNMSM (PERU), COMPARED WITH THE STUDENTS OF MANAGEMENT OF THE UNIVERSITY OF BERGAMO (ITALY)

Jorge Mayurí Barrón* jmayurib@unmsm.edu.pe
\end{abstract}

[RECEPCIÓN: AGOSTO 2015 / CONFORMIDAD: NOVIEMBRE 2015]

\section{RESUMEN}

La investigación se centra en un estudio comparativo del uso de la herramienta de marketing personal y la ventaja competitiva que genera esta herramienta entre alumnos de la Escuela Académica de Administración, Escuela Académica de Negocios Internacionales y Escuela Académica de Administración en Turismo de la Facultad de Ciencias Administrativas de la Universidad Nacional Mayor de San Marcos del Perú y los alumnos del Área de Gerencia de la Universidad de los Estudios de Bérgamo de Italia. Para ello se aplicaron encuestas a los alumnos de ambas universidades, determinándose que los alumnos de la universidad de Bérgamo usaban mejor el marketing personal y por ello, generaban una mejor ventaja competitiva personal con respecto a los alumnos de la universidad del Perú. El tipo de estudio realizado fue no experimental y transversal levantándose los datos en un solo momento para cada universidad

Palabras clave: Marketing personal, ventaja competitiva.

\footnotetext{
Profesor e Investigador asociado de la Facultad de Ciencias Administrativas, Universidad Nacional Mayor de San Marcos.
} 


\begin{abstract}
The research focuses on a comparative study of the use of the Personal Marketing tool and competitive advantage generated by this tool between students of the Academic School of Management, Academic School of International Business and Academic School of Tourism of the Faculty of Business Administration of the Major National University of San Marcos in Peru and students of the Department of Management at the University of Bergamo in Italy, this survey was conducted to students of both universities determining that the students of the University of Bergamo use best marketing staff and therefore generating a better personal competitive advantage over students from the University of Peru, the type of study not experimental, cross-section at one time for each university
\end{abstract}

Keywords: Personal marketing, competitive advantage.

\section{INTRODUCCIÓN}

El objetivo central de la investigación es demostrar que el llamado marketing personal, como herramienta de gestión, está viviendo cambios bastante radicales en su concepto y aplicación a la competitividad. Esto significa que el marketing personal requiere de una nueva concepción en la imagen, las actitudes, las relaciones interpersonales y las habilidades sociales de los alumnos de Marketing de las Escuelas Académico Profesionales de Administración, Administración de Negocios Internacionales y de Administración en Turismo de la Facultad de Ciencias Administrativas de la Universidad Nacional Mayor de San Marcos, si es que deseamos contar con egresados realmente competitivos.

La gestión del marketing personal se involucra en el desarrollo de las personas, y especialistas contemporáneos, los cuales consideran que este es el factor de la productividad y el progreso económico.

Para lograr mejores resultados en un ambiente de economía global, caracterizado por buenos competidores, las universidades deben modificar sus estrategias a fin de hacerse más efectivas para así orientarse hacia la construcción de la competencia, con la ayuda de la información de cada mercado.

\section{PLANTEAMIENTO DEL ESTUDIO}

El surgimiento de un recurso humano global y su adecuación a los nuevos tiempos constituye un reto para las Escuelas Académico Profesionales de Administración, Administración de Negocios Internacionales y de Administración en Turismo de la Facultad de Ciencias Administrativas de la Universidad Nacional Mayor de San Marcos, siendo necesario afrontar decididamente los escenarios de mercados globalizados, si es el deseo de permanecer en ellos siendo más competitivos. Es por ello que, ante nuevas estrategias empresariales, se hace indispensables el repensar, el diseñar, y ejecutar temas como el marketing personal como herramienta de gestión, que permita mejorar la gestión y organización de empresas competitivas, recordando que las ventajas competitivas son y serán siempre las personas.

\section{MARCO TEÓRICO}

El marketing personal implica, a nivel del empresariado, analizar los cuatro elementos que lo determinan, en un escenario de competitividad (Stanton, 2007).

En primer lugar tenemos a la comunicación personal. Es la forma en la cual nosotros hacemos llegar nuestros servicios al usuario final, esto lo hacemos en forma personal, de manera directa, o a través de diversos medios de comunicación como radio, televisión, prensa escrita, etcétera. Debemos tener un cuidado muy en especial de nuestra imagen ya que nuestro cuerpo comunica lo que somos o podemos ser.

La imagen es tan importante que existen empresas en las que los trabajadores visten de uniforme para mostrar un espíritu de equipo, una entidad grupal entre todas las personas que componen dicho proyecto de empresa. Por tanto, la clave a la hora de vestir de una forma adecuada para ir a trabajar reside en pensar no solo en los gustos personales, sino también en la normativa de la empresa sobre esta cuestión.

El segundo elemento del marketing personal es la distribución personal; es decir, la forma en la que hacemos llegar nuestro servicio. Por lo gene- 
ral, nosotros estamos físicamente, pero también hoy en día este servicio se brinda vía on line, por teléfono o cualquier otro medio de comunicación.

En tercer lugar tenemos el precio personal. Como profesionales, cuando brindamos un servicio, estamos intercambiando satisfactores, entregamos lo que el cliente desea, quien retribuye esto mediante un pago que en muchos casos suele ser en dinero.

El cuarto elemento de un buen marketing personal es el servicio personal, es en sí lo que estamos intercambiando; por ejemplo, el médico cirujano intercambia sus conocimientos y experiencia durante una operación, un docente de igual manera al impartir su cátedra.

El marketing personal como herramienta de gestión para la competitividad, requiere de:

- Contar con docentes no tradicionales en marketing, que brinden una nueva mezcla de marketing personal.

- Orientar a los alumnos a la competencia, pero orientados al proceso y a las utilidades.

- La transformación o impacto que se requiere derivado de los puntos antes mencionados, será el surgimiento de la economía de información, a la que habrá de ajustarse y de la cual será necesario aprender.

\section{OBJETIVOS ALCANZADOS}

Con relación a los objetivos alcanzados, se informa lo siguiente:

\section{Objetivo general}

Determinar de qué manera el marketing personal influye en la ventaja competitiva en los alumnos de la Facultad de Ciencias Administrativas de la Universidad Nacional Mayor de San Marcos del Perú comparada con los alumnos de Administración de la Universidad de los Estudios de Bérgamo (Università degli Studi di Bergamo), Italia.

Mediante la aplicación del Modelo de Regresión y Correlación se determinó la existencia de una relación directa y significativa de las variables marketing personal y competitividad en los alumnos de la Facultad de Ciencias Administrativas de la Universidad Nacional Mayor de San Marcos del Perú comparada con los alumnos de Administración de la Universidad de los Estudios de Bérgamo (Università degli Studi di Bergamo), Italia.

\section{Objetivos específicos}

- Objetivo específico 1. Evaluar de qué manera la comunicación en el marketing personal influye en la ventaja competitiva de los alumnos de la Facultad de Ciencias Administrativas de la Universidad Nacional Mayor de San Marcos del Perú comparada con los alumnos de Administración de la Universidad de los Estudios de Bérgamo (Università degli Studi di Bergamo), Italia.

Se determinó mediante la aplicación del Modelo de Regresión y Correlación la existencia de una relación directa y significativa de las variables comunicación en el marketing personal y la competitividad en los alumnos de la Facultad de Ciencias Administrativas de la Universidad Nacional Mayor de San Marcos del Perú comparada con los alumnos de Administración de la Universidad de los Estudios de Bérgamo (Università degli Studi di Bergamo), Italia.

- Objetivo específico 2. Evaluar de qué manera la distribución en el marketing personal influye en la ventaja competitiva de los alumnos de la Facultad de Ciencias Administrativas de la Universidad Nacional Mayor de San Marcos del Perú comparada con los alumnos de Administración de la Universidad de los Estudios de Bérgamo (Università degli Studi di Bergamo), Italia.

Se determinó mediante la aplicación del Modelo de Regresión y Correlación la existencia de una relación directa y significativa de las variables distribución en el marketing personal y la competitividad en los alumnos de la Facultad de Ciencias Administrativas de la Universidad Nacional Mayor de San Marcos del Perú comparada con los alumnos de Administración de la Universidad de los Estudios de Bérgamo (Università degli Studi di Bergamo), Italia.

- Objetivo específico 3. Evaluar de qué manera el precio en el marketing personal influye en la ventaja competitiva en los alumnos de la Facultad de Ciencias Administrativas de la Universidad Nacional Mayor de San Marcos del Perú comparada con los alumnos de Administración de la Universidad de los Estudios de Bérgamo (Università degli Studi di Bergamo), Italia. 
Se determinó mediante la aplicación del Modelo de Regresión y Correlación la existencia de una relación directa y significativa de la variable precio en el marketing personal y la competitividad, en los alumnos de la Facultad de Ciencias Administrativas de la Universidad Nacional Mayor de San Marcos del Perú comparada con los alumnos de Administración de la Universidad de los Estudios de Bérgamo (Università degli Studi di Bergamo), Italia.

- Objetivo específico 4. Evaluar de qué manera el servicio en el marketing personal influye en la ventaja competitiva en los alumnos de la Facultad de Ciencias Administrativas de la Universidad Nacional Mayor de San Marcos del Perú comparada con los alumnos de Administración de la Universidad de los Estudios de Bérgamo (Università degli Studi di Bergamo), Italia.

Se determinó mediante la aplicación del Modelo de Regresión y Correlación la existencia de una relación directa y significativa de la variable servicio en el marketing personal y la competitividad, en los alumnos de la Facultad de Ciencias Administrativas de la Universidad Nacional Mayor de San Marcos del Perú comparada con los alumnos de Administración de la Universidad de los Estudios de Bérgamo (Università degli Studi di Bergamo), Italia.

\section{HIPÓTESIS DEMOSTRADA}

Se formuló la siguiente hipótesis de trabajo general:

- Ho: El marketing personal no influye en la ventaja competitiva en los alumnos de la Facultad de Ciencias Administrativas de la Universidad Nacional Mayor de San Marcos del Perú comparada con los alumnos de Administración de la Universidad de los Estudios de Bérgamo (Università degli Studi di Bergamo), Italia.

- Ha: El marketing personal influye en la ventaja competitiva en los alumnos de la Facultad de Ciencias Administrativas de la Universidad Nacional Mayor de San Marcos del Perú comparada con los alumnos de Administración de la Universidad de los Estudios de Bérgamo (Università degli Studi di Bergamo), Italia.

- Ho1: La comunicación en el marketing personal no influye en la ventaja competitiva en los alumnos de la Facultad de Ciencias Administra- tivas de la Universidad Nacional Mayor de San Marcos del Perú comparada con los alumnos de Administración de la Universidad de los Estudios de Bérgamo (Università degli Studi di Bergamo), Italia.

- Ha1: La comunicación en el marketing personal influye en la ventaja competitiva en los alumnos de la Facultad de Ciencias Administrativas de la Universidad Nacional Mayor de San Marcos del Perú comparada con los alumnos de Administración de la Universidad de los Estudios de Bérgamo (Università degli Studi di Bergamo), Italia.

- Ho2: La distribución en el marketing personal no influye en la ventaja competitiva en los alumnos de la Facultad de Ciencias Administrativas de la Universidad Nacional Mayor de San Marcos del Perú comparada con los alumnos de Administración de la Universidad de los Estudios de Bérgamo (Università degli Studi di Bergamo), Italia.

- Ha2: La distribución en el marketing personal influye en la ventaja competitiva en los alumnos de la Facultad de Ciencias Administrativas de la Universidad Nacional Mayor de San Marcos del Perú comparada con los alumnos de Administración de la Universidad de los Estudios de Bérgamo (Università degli Studi di Bergamo), Italia.

- Ho3: El precio en el marketing personal no influye en la ventaja competitiva en los alumnos de la Facultad de Ciencias Administrativas de la Universidad Nacional Mayor de San Marcos del Perú comparada con los alumnos de Administración de la Universidad de los Estudios de Bérgamo (Università degli Studi di Bergamo), Italia.

- Ha3: El precio en el marketing personal influye en la ventaja competitiva en los alumnos de la Facultad de Ciencias Administrativas de la Universidad Nacional Mayor de San Marcos del Perú comparada con los alumnos de Administración de la Universidad de los Estudios de Bérgamo (Università degli Studi di Bergamo), Italia.

- Ho4: El servicio en el marketing personal no influye en la ventaja competitiva en los alumnos de la Facultad de Ciencias Administrativas de la Universidad Nacional Mayor de San Marcos del Perú comparada con los alumnos 
de Administración de la Universidad de los Estudios de Bérgamo (Università degli Studi di Bergamo), Italia.

- Ha4: El servicio en el marketing personal influye en la ventaja competitiva en los alumnos de la Facultad de Ciencias Administrativas de la Universidad Nacional Mayor de San Marcos del Perú comparada con los alumnos de Administración de la Universidad de los Estudios de Bérgamo (Università degli Studi di Bergamo), Italia.

\section{METODOLOGÍA Y TÉCNICAS DE INVESTIGACIÓN UTILIZADAS}

\section{Metodología}

Según Sierra Bravo (1994) la investigación científica social es un proceso de aplicación del método y técnicas científicas, en la cual, según el criterio del investigador, se pueden adoptar los tipos de investigación básica (o teórica), y la aplicada. La investigación básica permite explicar el funcionamiento y conocimiento de la infraestructura de los fenómenos sociales.

De acuerdo a Sierra Bravo (1994) la investigación es de tipo básico, pues los métodos a ejecutar están orientados a recoger información de la realidad para enriquecer los conocimientos y la aplicación del marketing personal y competitividad en la Facultad de Ciencias Administrativas de la Universidad Nacional Mayor de San Marcos.

\section{Diseño de investigación}

Según Hernández (2010), el diseño de investigación es no experimental, de corte transversal de acuerdo a las siguientes consideraciones:

- Diseño No Experimental. Se denomina así por cuanto no se manipula el factor causal (variable independiente) para la determinación posterior de sus efectos. Solo se describe y se analiza su incidencia e interrelación en un momento dado de las variables.

- Diseño transversal. Se denomina así porque los objetivos generales y específicos están dirigidos al análisis del nivel o estado de las variables mediante la recolección de datos en un punto en el tiempo.

En la Figura $\mathrm{N}^{\circ} 1$ se muestran los resultados comparados del marketing y la ventaja competitiva en los alumnos de la Facultad de Ciencias Administrativas de la Universidad Nacional Mayor de San Marcos del Perú comparada con los alumnos de Administración de la Universidad de los estudios de Bérgamo (Università degli Studi di Bergamo), Italia.

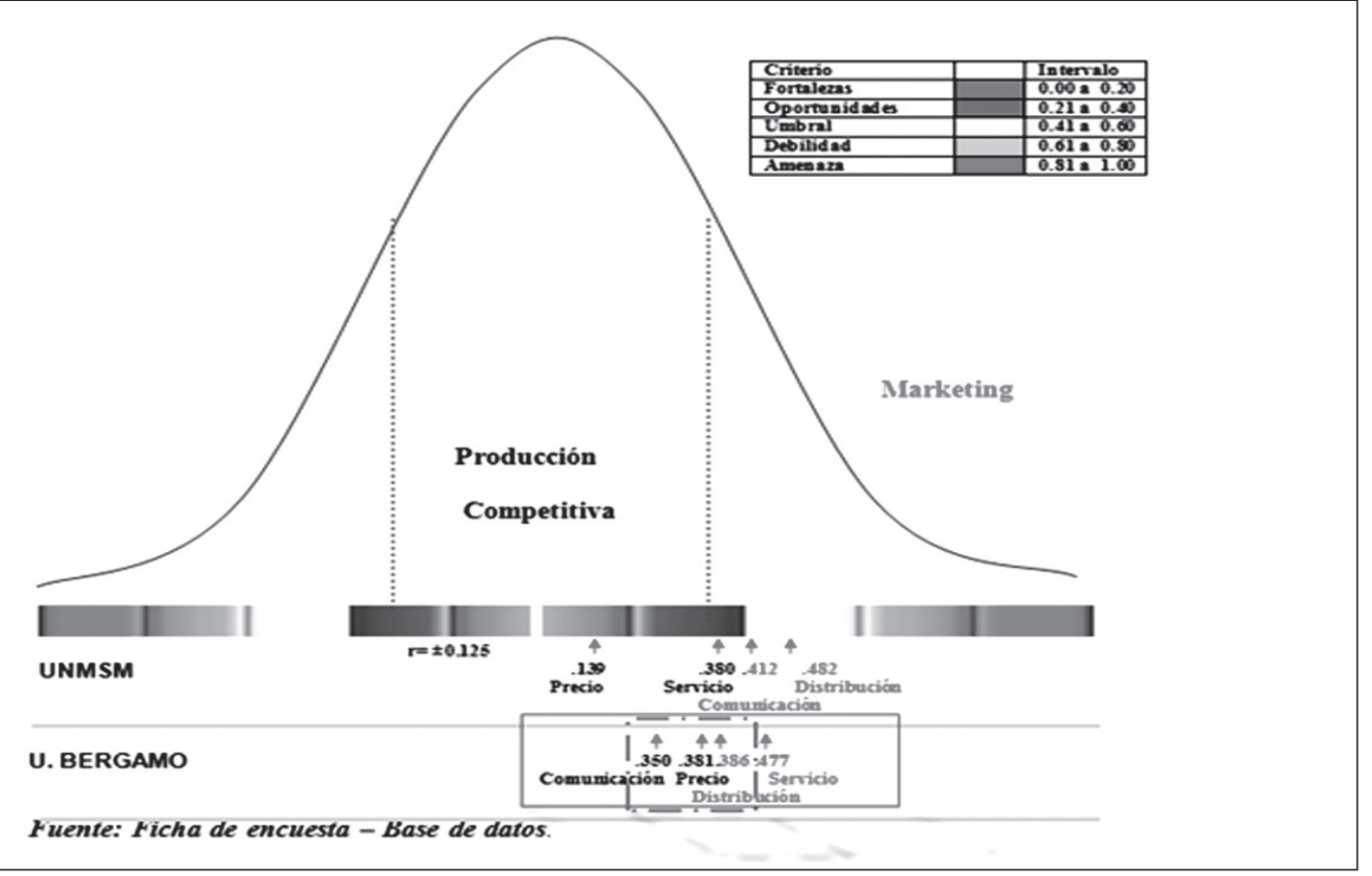

Fig. № 1 . Influencia del marketing y la ventaja competitiva en los alumnos de la Facultad de Ciencias Administrativas de la Universidad Nacional Mayor de San Marcos del Perú comparada con los alumnos de Administración de la Universidad de los estudios de Bérgamo (Università degli Studi di Bergamo), Italia. 


\section{Técnicas de investigación}

La técnica utilizada para la recolección de datos fue la aplicación de una encuesta anónima a la cual se le aplicó la validez de contenido, validada inicialmente por juicio de expertos, y cuyos resultados correlacionados se muestran en la Figura $\mathrm{N}^{\circ} 1$.

La presente investigación se viene realizando a la totalidad de los alumnos de la Facultad de Ciencias Administrativas de la Universidad Nacional Mayor de San Marcos del Perú comparada con los alumnos de Administración de la Universidad de los estudios de Bérgamo (Università degli Studi di Bergamo), Italia, con una muestra total de 90 encuestas.

\begin{tabular}{|c|c|c|}
\hline UNIVERSIDADES & Población & Muestra \\
\hline UNMSM & 1,235 & 45 \\
\hline U. BERGAMO & 1,300 & 45 \\
\hline TOTAL & 2,535 & 90 \\
\hline
\end{tabular}

\section{Recolección e interpretación de datos}

La recolección y el procesamiento de datos se realizó utilizando el software SPSS en su versión 22; y la interpretación de los datos requirió la utilización del criterio del valor "p" para el contraste de hipótesis.

\section{RESULTADOS Y DISCUSIÓN}

La hipótesis requirió previamente del siguiente criterio estadístico de contraste de hipótesis:

- Se realizó mediante el criterio teórico del valor $\mathrm{p}=0.05$.

- Si en la colecta y procesamiento de los datos se obtiene en su cálculo un valor $p \geq 0.05$, se aceptará la hipótesis nula (Ho). Pero si en la colecta y procesamiento de los datos se obtiene en su cálculo un valor $\mathrm{p} \leq 0.05$, se aceptará la hipótesis alternativa (Ha).

- Utilizar la tabla de correlaciones y gráfica según método de la cuarta vía (Sánchez, 2011).

\begin{tabular}{|l|l|l|}
\hline Criterio & Intervalo \\
\hline Fortalezas & 0.00 a 0.20 \\
\hline Oportunidades & 0.21 a 0.40 \\
\hline Umbral & & 0.41 a 0.60 \\
\hline Debilidad & 0.61 a 0.80 \\
\hline Amenaza & 0.81 a 1.00 \\
\hline
\end{tabular}

\section{Contrastación de hipótesis}

Se utilizaron tres criterios:

Se utilizó el criterio estadístico del Modelo de regresión y Correlación para determinar, en primer lugar, la correlación conjunta de ambas variables $\mathrm{y}$, en segundo lugar, las correlaciones parciales de las dimensiones de la variable independiente con la variable dependiente, por universidad.

Como producto de cada Modelo de Regresión y Correlación, por universidad, se determinó mediante la aplicación de los valores hallados por la distribución Beta, qué dimensiones del marketing personal son las que más influyen (explican) las diferencias por universidad.

Se determinó la correlación de las variables marketing personal y competitividad en los alumnos de la Facultad de Ciencias Administrativas de la Universidad Nacional Mayor de San Marcos del Perú, y se halló una correlación directa y significativa de 0.517 ; $\mathrm{y}$ un valor $\mathrm{p}=0.000$.

\section{Cuadro $\mathrm{N}^{\circ} 1$}

\begin{tabular}{|c|c|c|c|c|c|}
\hline Modelo & $\mathrm{R}$ & $\begin{array}{c}\mathrm{R} \\
\text { cuadrado }\end{array}$ & $\begin{array}{c}\mathrm{R} \text { cuadrado } \\
\text { corregida }\end{array}$ & $\begin{array}{c}\text { Error típico de } \\
\text { la estimación }\end{array}$ & Valor $\mathrm{p}$ \\
\hline .1 &, $517 \mathrm{a}$ &, 267 &, 256 &, 000 & .000 \\
\hline
\end{tabular}

Mediante la utilización de la Distribución Beta, se determinó que las dimensiones del marketing personal, por la Facultad de Ciencias Administrativas de la Universidad Nacional Mayor de San Marcos del Perú que influyen en el nivel umbral en la competitividad son la distribución personal $(\mathrm{r}=0.482 ; \mathrm{p}=0.000)$, y comunicación personal ( $\mathrm{r}$ $=0.448 ; p=0.000$ ). La que influye en el nivel de oportunidad en la competitividad es el servicio personal $(r=0.380 ; p=0.000)$, y con nivel fortaleza, el precio personal $(r=0.139 ; p=0.000)$.

\section{Cuadro $\mathrm{N}^{\circ} 2$}

\begin{tabular}{|c|c|c|c|c|c|}
\hline \multirow{2}{*}{$\begin{array}{c}\text { Modelo UNMSM } \\
\text { B }\end{array}$} & \multicolumn{2}{|c|}{$\begin{array}{l}\text { Coeficientes no } \\
\text { estandarizados }\end{array}$} & \multirow[t]{2}{*}{$\begin{array}{l}\text { Coeficientes } \\
\text { tipificados }\end{array}$} & \multirow{2}{*}{$\begin{array}{c}\text { Valor } \\
p\end{array}$} & \multirow{2}{*}{$\begin{array}{c}\text { Correlación } \\
\text { parcial }\end{array}$} \\
\hline & $\begin{array}{l}\text { Error } \\
\text { típico }\end{array}$ & Beta & & & \\
\hline (Constante) &,- 266 & ,326 & & & \\
\hline $\begin{array}{c}\text { Distribución } \\
\text { personal }\end{array}$ & ,468 & ,072 & ,324 & ,000 & ,482 \\
\hline Precio personal &,- 026 & 071 &,- 017 &, 000 & ,139 \\
\hline Servicio personal & 188 & ,047 & , 191 &, 000 & ,380 \\
\hline $\begin{array}{c}\text { Comunicación } \\
\text { personal }\end{array}$ & ,354 &, 079 & ,228 &, 000 & ,412 \\
\hline
\end{tabular}


Se determinó luego, la correlación de las variables marketing personal y competitividad en los alumnos de Administración de la Universidad de los Estudios de Bérgamo (Università degli Studi di Bergamo), Italia. Se halló una correlación directa y significativa de 0.480 ; y un valor $\mathrm{p}=0.000$.

\section{Cuadro $\mathrm{N}^{\circ} 3$}

\begin{tabular}{|c|c|c|c|c|c|}
\hline Modelo & $\mathrm{R}$ & $\begin{array}{c}\text { R cuadra- } \\
\text { do }\end{array}$ & $\begin{array}{c}\text { R cuadra- } \\
\text { do cor- } \\
\text { regida }\end{array}$ & $\begin{array}{c}\text { Error típico } \\
\text { de la esti- } \\
\text { mación }\end{array}$ & Valor $p$ \\
\hline .1 &, $480 \mathrm{a}$ &, 231 &, 219 &, 37704 &, 000 \\
\hline
\end{tabular}

Mediante la utilización de la distribución Beta se determinó que las dimensiones del marketing personal que influyen en el nivel umbral en la competitividad es el servicio personal $(r=0.477$; $\mathrm{p}=0.000$ ). En un nivel de oportunidades se hallan la comunicación personal $(\mathrm{r}=0.350 ; \mathrm{p}=0.000)$, la distribución personal $(\mathrm{r}=0.386 ; \mathrm{p}=0.000), \mathrm{y}$ el precio personal $(r=0.381 ; p=0.000)$.

\section{Cuadro $\mathrm{N}^{\circ} 4$}

\begin{tabular}{|c|c|c|c|c|c|c|}
\hline \multicolumn{2}{|c|}{$\begin{array}{c}\text { Modelo U. BER- } \\
\text { GAMO }\end{array}$} & \multicolumn{2}{|c|}{$\begin{array}{l}\text { Coeficientes no } \\
\text { estandarizados }\end{array}$} & \multirow[t]{2}{*}{$\begin{array}{c}\text { Coeficientes } \\
\text { tipificados }\end{array}$} & \multirow{2}{*}{$\begin{array}{c}\text { Valor } \\
p\end{array}$} & \multirow{2}{*}{$\begin{array}{c}\text { Correlación } \\
\text { parcial }\end{array}$} \\
\hline & B & $\begin{array}{l}\text { Error } \\
\text { típico }\end{array}$ & Beta & & & \\
\hline \multirow{5}{*}{1} & (Constante) & 2,648 & ,075 & & & \\
\hline & $\begin{array}{l}\text { Distribución } \\
\text { Personal }\end{array}$ & ,078 & ,023 & ,197 & .000 & ,386 \\
\hline & $\begin{array}{l}\text { Precio } \\
\text { Personal }\end{array}$ & ,040 & ,021 & ,108 & ,000 & ,381 \\
\hline & $\begin{array}{l}\text { Servicio } \\
\text { Personal } \\
\end{array}$ & ,139 & ,022 & ,350 & .000 & ,477 \\
\hline & $\begin{array}{c}\text { Comu- } \\
\text { nicación } \\
\text { Personal }\end{array}$ & ,013 & ,024 & ,032 & ,000 & ,350 \\
\hline & & & & & & \\
\hline
\end{tabular}

\section{CONCLUSIONES}

\section{Conclusión general}

Se determinó mediante la aplicación del Modelo de Regresión y Correlación la existencia de una relación directa y significativa de las variables marketing personal y competitividad, en los alumnos de la Facultad de Ciencias Administrativas de la Universidad Nacional Mayor de San Marcos del Perú comparada con los alumnos de Administración de la Universidad de los Estudios de Bérgamo (Università degli Studi di Bergamo), Italia.

1. Conclusión específica 1. Se determinó mediante la aplicación del Modelo de Regresión y Correlación la existencia de una relación directa y significativa de la dimensión precio personal y competitividad en los alumnos de la Facultad de Ciencias Administrativas de la Universidad Nacional Mayor de San Marcos del Perú comparada con los alumnos de Administración de la Universidad de los Estudios de Bérgamo (Università degli Studi di Bergamo), Italia.

2. Conclusión específica 2. Se determinó mediante la aplicación del Modelo de Regresión y Correlación la existencia de una relación directa y significativa de la dimensión servicio personal y competitividad en los alumnos de la Facultad de Ciencias Administrativas de la Universidad Nacional Mayor de San Marcos del Perú comparada con los alumnos de Administración de la Universidad de los Estudios de Bérgamo (Università degli Studi di Bergamo), Italia.

3. Conclusión específica 3. Se determinó mediante la aplicación del Modelo de Regresión y Correlación la existencia de una relación directa y significativa de la dimensión comunicación personal y competitividad en los alumnos de la Facultad de Ciencias Administrativas de la Universidad Nacional Mayor de San Marcos del Perú comparada con los alumnos de Administración de la Universidad de los Estudios de Bérgamo (Università degli Studi di Bergamo), Italia.

4. Conclusión específica 4. Se determinó mediante la aplicación del Modelo de Regresión y Correlación la existencia de una relación directa y significativa de la dimensión distribución personal y competitividad en los alumnos de la Facultad de Ciencias Administrativas de la Universidad Nacional Mayor de San Marcos del Perú comparada con los alumnos de Administración de la Universidad de los Estudios de Bérgamo (Università degli Studi di Bergamo), Italia.

\section{RECOMENDACIONES}

- Cambiar la gestión del marketing personal, realinearla como una función administrativa y volverla más estratégica según las posiciones, laboral, social y económica.

- La transformación deseada involucra -principalmente- compromiso, trabajo en equipo y liderazgo efectivo. 


\section{REFERENCIAS BIBLIOGRÁFICAS}

González Vásquez, Encarnación, (2005). Casos De Dirección De Marketing, Madrid: Pearson.

Hernández S., (2010): Metodología de la Investigación. Cuarta edición, Mc Graw Hill, México D.F.

Hoffman, K. Douglas, (2007). Principios De Marketing y Sus Mejores Prácticas. México, D. F.: Thomson.

Kotler, Philip (2004). Marketing. Madrid, Pearson.

Kotler, Philip, (2007). Marketing. México, D.F., Prentice Hall.

Kotler, Philip. (2008). Fundamentos De Marketing, México, D.F., Pearson Educación.

Mayorga, David (2005). Marketing Estratégico. En: La Empresa Peruana. Lima, Universidad del Pacífico, Centro de Investigación.
Mullins, John W. (2007). Administración Del Marketing, México, D.F., McGraw-Hill Interamericana.

Rico, Rubén Roberto (2008). Export Marketing. Buenos Aires, Prentice Hall.

Sierra Bravo, R. (1994). Técnicas de investigación social. Madrid. Novena edición, Ed. Paraninfo.

Stanton, W. (2007). Fundamentos de Marketing. México. D.F.Décimo cuarta edición. Mc Graw Hill.

Stanton, William J. (2007). Fundamentos De Marketing, México, D.F., McGraw-Hill

Walker, Orville C. (2005). Marketing Estratégico, México, D.F., McGraw-Hill. 